\title{
METABOLIC INVESTIGATION OF PATIENTS WITH UROLITHIASIS IN A SPECIFIC REGION
}

\author{
LUIS A. B. PERES, ANDRÉ S. MOLINA, MARCOS H.L. GALLES \\ State University of West Paraná, UNIOESTE, Cascavel, Paraná, Brazil
}

\begin{abstract}
Objective: To assess the prevalence of the main metabolic alterations found in patients with recent diagnosis of urolithiasis in the West region of Paraná state, Brazil.

Materials and Methods: We made a retrospective study on 425 patients with evidence of recent formation of renal stones. Laboratory assessment consisted in 3 samples of 24-hour urine with dosing of calcium, uric acid, citrate, oxalate, sodium and creatinine. A urine culture was also made and qualitative cystinuria and urinary $\mathrm{pH}$ following 12-hour fasting and water restriction were evaluated.

Results: In $96.5 \%$ of patients a cause was detected for the urolithiasis. Metabolic alterations most frequently found were: hypercalciuria (38.3\%), hypocitraturia (29.6\%) and hyperexcretion of uric acid $(21.6 \%)$. Low urinary volume $(17.9 \%)$, urinary tract infection $(12.9 \%)$, hyperparathyroidism $(3.3 \%)$, renal tubular acidosis $(1.2 \%)$, cystinuria $(0.9 \%)$ and anatomical alterations $(12.7 \%)$ were also observed.

Conclusions: Hypercalciuria, hypocitraturia and hyperuricuria are the most frequent metabolic disorders in the population under study and these data are in accordance to the literature.
\end{abstract}

Key words: urolithiasis; metabolism; metabolic disease; risk factors; calcium oxalate; calcium Int Braz J Urol. 2003; 29: 217-220

\section{INTRODUCTION}

Renal lithiasis affects $12 \%$ of the United States of America population and its recurrence can reach $50 \%$. Significant advancements in surgical approach have occurred, but they did not change the natural history of urolithiasis $(1,2)$. In Brazil there is a report that $5 \%$ of the population has urolithiasis, which corresponds to more than 7 million of patients with lithiasis. It is a pathology that affects young people, with its peak incidence during the third decade of life and a very high recurrence, reducing productivity of such individuals (3). The dissemination of preventive metabolic investigation programs and nephrologic approach is necessary, however the high costs of laboratory tests and the poor adhesion of pa- tients to treatment and to dietary orientation limit the success of the medical approach.

The objective of the present work is to show the prevalence of major metabolic alterations found in patients with renal lithiasis coming from urban and rural zones of West region of Paraná state, Brazil.

\section{MATERIALS AND METHODS}

It was performed a retrospective study on 425 patients from general nephrology outpatient service coming from rural and urban zones who presented evidences of recent urolithiasis, in the period from 1995 to 2002. Inclusion criteria for patients in this retrospective study were spontaneous, endoscopic or surgical elimination of stones and/or radiological con- 
firmation of presence of stones in urinary tract in the past 6 months.

Laboratory investigation consisted in 3 blood and 24-hour urine samples, with a 30-day interval between them. Calcium, uric acid, citrate, sodium, creatinine and oxalate were evaluated in 24-hour urine, and calcium, uric acid, creatinine and parathormone in blood. Qualitative cystinuria, urinary $\mathrm{pH}$ following a 12-hour fasting and water restriction and urine culture were also performed.

General nutritional orientations were offered to all patients following the second samples collection, consisting in: 6 grams of salt/day, 1 gram $/ \mathrm{Kg}$ of weight of proteins, restriction of purines to $150 \mathrm{mg}$, adjustment of calcium intake to $1000 \mathrm{mg}$ and increase in fluid intake to maintain a urinary volume above 20 $\mathrm{mL} / \mathrm{kg} /$ day.

Laboratory methods employed and reference values adopted for 24-hour urine samples were: calcium - atomic absorption spectrophotometry method $(<4.0 \mathrm{mg} / \mathrm{kg}$ ), uric acid - uricase enzymatic method (up to $750 \mathrm{mg}$ for females and $800 \mathrm{mg}$ for males), citrate - citrate-lyase enzymatic method (>320 mg), sodium - selective ion method $(<150 \mathrm{mEq})$, creatinine - alkaline picrate method (>1000 mg) and urinary volume - volumetric measurement in Becker by visual analysis. For plasma dosing the methods employed were: calcium - colorimetric method ( 8.5 - $10.5 \mathrm{mg} / \mathrm{dL}$ ), uric acid - uricase colorimetric method (2.0 to $7.0 \mathrm{mg} / \mathrm{dL})$, creatinine - alkaline picrate method $(0.7$ a $1.4 \mathrm{mg} / \mathrm{dL})$ e parathormone intact molecule assay. For assays in single urine sample the methods were: qualitative cystinuria sodium nitroprusside test, and urinary $\mathrm{pH}$ - measurement by reactive strips with methyl red and bromthymol blue indicator system. Urinary volume was considered to be decreased, when at least one of the samples presented a 24-hour urinary volume lower than $15 \mathrm{~mL} / \mathrm{kg}$.

\section{RESULTS}

1,023 patients with lithiasis were assessed out of a total of 5,207 attended patients, among whom only 425 concluded the metabolic study. Patients mean age was 32.2 years (ranging from 2 months to 67 years), $61.2 \%$ were male and $38.8 \%$ were female. As for race, $85 \%$ were Caucasian.

Among the 425 patients with lithiasis under study, at least one alteration was found in 410 (96.5\%). 604 diagnoses were made, showing that some patients present more than one alteration. Metabolic alterations found were: hypercalciuria in $38.3 \%$, hypocitraturia in $29.6 \%$, hyperexcretion of uric acid in $21.6 \%$, low urinary volume in $17.9 \%$, urinary tract infection in $12.9 \%$, hyperparathyroidism in $3.3 \%$, renal tubular acidosis in $1.2 \%$ and cystinuria in $0.9 \%$ (Table-1). Anatomical alterations were found in $12.7 \%$. In $3.5 \%$ of patients no alterations were found.

Among the anatomical alterations found (Table-2), we observed 22 renal cysts $(40.7 \%)$, pyelocaliceal duplications $(20.4 \%), 5$ atrophic kidneys $(9.3 \%), 5$ stenoses of ureteropelvic junction UPJ (9.3\%), 5 single kidneys (9.3\%), 3 neurogenic bladders $(5.5 \%), 1$ pelvic kidney (1.8\%), 1 horseshoe kidney $(1.8 \%)$ and 1 medullary sponge kidney $(1.8 \%)$.

\section{DISCUSSION}

Nephrolithiasis is a disease with high prevalence and recurrence, being one of the most common diseases of the urinary tract (4). We do not have available data about prevalence of renal lithiasis in the general population in the West region of Paraná state, Brazil. In this study we observed that approximately

Table 1 - Metabolic alterations found.

\begin{tabular}{lrc}
\hline Metabolic Alteration & N & \% \\
\hline Hypercalciuria & 163 & 29.6 \\
Hypocitraturia & 126 & 22.9 \\
Hyperuricuria & 92 & 16.7 \\
Low urinary volume & 76 & 13.8 \\
Urinary tract infection & 55 & 10.0 \\
Hyperparathyroidism & 14 & 2.5 \\
Renal tubular acidosis & 5 & 0.9 \\
Cystinuria & 4 & 0.7 \\
No alteration detected & 15 & 2.7 \\
Total & 550 & 100 \\
\hline
\end{tabular}


Table 2 - Anatomical alterations found in $12.7 \%$ of patients with urolithiasis.

\begin{tabular}{lrc}
\hline Anatomical Alteration & N & \% \\
\hline Renal cyst & 22 & 40.7 \\
Pyelocaliceal duplication & 11 & 20.4 \\
Atrophic kidney & 5 & 9.3 \\
Ureteropelvic junction stenosis & 5 & 9.3 \\
Single kidney & 5 & 9.3 \\
Neurogenic bladder & 3 & 5.5 \\
Pelvic kidney & 1 & 1.8 \\
Horseshoe kidney & 1 & 1.8 \\
Medullary sponge kidney & 1 & 1.8 \\
Total & 54 & 100 \\
\hline
\end{tabular}

$20 \%$ of attended patients in a general nephrologic outpatient service, which receives patients coming from urban and rural zones, have the diagnosis of urolithiasis. The risk of terminal chronic renal insufficiency (CRI) is small in theses patients, but some conditions, if left untreated, present a high risk of evolving to renal failure (cystinuria, hyperparathyroidism, oxaluria, etc.). The prompt recognition of such conditions is important to prevent CRI (5).

Urolithiasis affects preferably young males (6). In our study there was a predominance of male gender $(61.2 \%)$ and patients mean age was 32.2 years, what is in accordance to the literature (7). There was a predominance of Caucasian race $(85 \%)$, reflecting the racial distribution in our region, and it does not enable us to say that incidence is higher in this race.

We detected a causal alteration in $96.5 \%$ of patients. Metabolic alterations most frequently found were hypercalciuria (29.6\%), hypocitraturia (22.9\%) and hyperexcretion of uric acid $(16.7 \%)$, data that is consonant with the majority of works, and it shows that there is a lack of balance between the promoters of stone formation and its inhibitors $(7,8)$.

Decrease in urinary volume is considered a cause of lithiasis (9). In hot climate countries extrarenal losses and low fluids intake can contribute to stone formation. In this study we observed a decreased urinary volume in $13.8 \%$ of alterations, a much lower index than the $77 \%$ index reported in the interior of São Paulo state, where climate is warmer (7).

Hypercalciuria is responsible for more than $50 \%$ of metabolic disorders in adults and 53 to $75 \%$ in children (10). It is thought to have a strong genetic component, probably with dominant autossomic inheritance (11). A sodium-rich diet is one factor to be considered in pathogenesis of hypercalciuria (12). In this study hypercalciuria was the prevalent metabolic disorder. In West region of Paraná state the ingestion of milk and dairy products is small, but salt and protein intake is high, probably contributing to occurrence of hypercalciuria. Oral calcium overload test was not routinely performed due to technical implications of the method and its cost, therefore patients were not classified according to type of hypercalciuria (renal or intestinal), because we understood that this classification does not change significantly the treatment of a patient who has hypercalciuria.

Hypocitraturia is found in about $30 \%$ of patients with lithiasis (13). In this study we observed this alteration in $22.9 \%$ of metabolic disorders. Hyperuricuria is due to a high intake of purines or an elevated endogenous production. Low intake of water and urinary $\mathrm{pH}<5.5$ favor the precipitation of uric acid (14). Hyperuricuria was evidenced in $16.7 \%$ of metabolic disorders in our patients, and we believe that the high regional protein intake is a risk factor. National literature observes this disorder, from $18 \%$ to $76 \%$ (7).

Hyperoxaluria is a rare disorder, and is found in approximately $1 \%$ of individuals under study (2), and it was not routinely investigated in our study. We understand that the lack of oxalate dosing in 24-hour urine did not compromise the diagnosis of the metabolic disorders reported here.

We believe that the right approach to theses disorders with a multidisciplinary team can reduce both incidence and recurrence of urolithiasis in our population.

This work served as a base for knowing the metabolic profile of lithiasis patients from West region of Paraná state, Brazil. The most frequent metabolic alterations were hypercalciuria, hypocitraturia and hyperuricuria. 


\section{REFERENCES}

1. Pearle MS: Prevention of nephrolithiasis. Curr Opin Nephrol Hypertens. 2001; 10: 203-9.

2. Wilkinson $\mathrm{H}$ : Clinical investigation and management of patients with renal stones. Ann Clin Biochem. 2001; 38: 180-7.

3. Sakuno MLD, Akimoto LS, Mereles EAL, Modenuti MI, Vieira AGM, Dal Col SMD, et al.: Contribution of the laboratory for clinical analysis for the metabolic diagnosis of renal lithiasis. Ver Bras Anal Clin. 1994; 26: 77-80 [in Portuguese].

4. Giugliani MCK: Metabolic Evaluation of Renal Lithiasis, Study in 100 Patients. Master of Science Thesis, Federal University of Rio Grande do Sul, 1990 [in Portuguese].

5. Gambaro G, Favaro S, D’Angelo A: Risk for renal failure in nephrolithiasis. Am J Kid Dis. 2001; 37: 233-43.

6. Robertson WG, Peacock M, Baker M, Marshall DH, Pearlman B, Speed R, et al.: Studies on the prevalence and epidemiology of urinary stone disease in men in Leeds. Br J Urol. 1983; 55: 595-8.
7. Ayusso LL, Schor N: Evaluation of patients with renal lithiasis in tropical region. Br J Nefrol. 2001; 23: 20512.

8. Tostes V, Cardoso LR: Recent advances in urolithiasis. Br J Nefrol. 2001; 23: 166-73 [in Portuguese].

9. Frank M, De Vries A, Lazebnik J, Kochwa S: Epidemiological investigation of urolithiasis in Israel. J Urol. 1959; 81: 497-502.

10. Levy FL, Adams-Huet B, Pak CYC: Ambulatory evaluation of nephrolithiasis: an update of a 1980 protocol. Am J Med. 1995; 98: 50-8.

11. Coe FL, Parks JH, Moore ES: Familial idiopathic hypercalciuria. N Engl J Med. 1979; 300: 337-40.

12. Pak CYC, Resnick MI: Medical therapy and new approaches to management of urolithiasis. Urol Clin North Am. 2000; 27: 243-53.

13. Pak CYC: Etiology and treatment of urolithiasis. Am J Kidney Dis. 1991; 18: 624-37.

14. Low RK, Stoller ML: Uric acid related nephrolithiasis. Urol Clin North Am. 1997; 24: 135-49.

Received: March 11, 2003

Accepted after revision: May 19, 2003

Correspondence address:

Dr. Luis Alberto Batista Peres

Rua São Paulo, 769 / 901

Cascavel, Paraná, 85801-020, Brazil

Fax: + 5545 327-3413

E-mail:peres@certto.com.br 